\title{
Conditions for the Implementation of a Patient Education Program Dedicated to Cancer Patients Treated by Oral Anticancer Therapy
}

This article was published in the following Dove Press journal: Patient Preference and Adherence

\author{
Elise Verot iD ' \\ Claire Falandry (iD ${ }^{2}$ \\ Véronique Régnier Denois (D) \\ Corinne Feutrier ${ }^{3}$ \\ Boris Chapoton' \\ Jean Okala (iD ${ }^{4}$ \\ Sidonie Pupier ${ }^{4}$ \\ Vanessa Rousset ${ }^{4}$ \\ Françoise Bridet ${ }^{3}$ \\ Christine Ravot ${ }^{2}$ \\ Catherine Rioufol ${ }^{5}$ \\ Véronique Trillet-Lenoir ${ }^{6}$ \\ Magali Hureau ${ }^{7}$ \\ Franck Chauvin' \\ Aurélie Bourmaud ${ }^{8}$
}

'University of Lyon, University of SaintEtienne, Centre Hygée, HESPER EA 7425,

Rue de la Marandière, Saint-Priest-en- Jarez, 42270, France; ${ }^{2}$ Oncogeriatrics Department, Lyon Sud Teaching Hospital \& Claude Bernard University, Lyon, France;

${ }^{3}$ Transversal Unit of Patient Education of Department of Rhône, Hospices Civils de Lyon, Lyon 69004, France; ${ }^{4}$ Public Health Department, Centre Hygée, Institut de Cancérologie Lucien Neuwirth, HESPER EA

7425, Saint-Priest-en-Jarez 42270, France;

${ }^{5}$ Pharmacy Department, Lyon Sud Teaching Hospital \& Claude Bernard University, Lyon, France; ${ }^{6}$ Medical Oncology Department, Lyon Sud Teaching Hospital \& Claude Bernard University, Lyon, France; ${ }^{7}$ Department of Clinical Research and Innovation, Léon Bérard Cancer Centre, Lyon, France; ${ }^{8}$ Clinical Epidemiology Unit, Robert Debré Hospital, AP-HP, INSERM CIC-EC I426; INSERM I I 23 ECEVE, University of Paris, Paris, France

Correspondence: Elise Verot University of Lyon, University of SaintEtienne, Centre Hygée, HESPER EA 7425, Rue de la Marandière, Saint-Etienne 42270, France

Tel +33-682309796

Email elise.verot@univ-st-etienne.fr
Introduction: A patient education program has been developed in the field of cancer for supporting cancer patients undergoing oral anticancer therapies. Its implementation was tested in 3 different settings. The objectives of this study were to 1) identify barriers and facilitators for implementing the patient education program, 2) identify practices encouraging or hindering implementation and 3) produce recommendations for its dissemination.

Methods: Twenty semi-structured interviews were conducted with caregivers from all three establishments.

Results: The main factors associated with successful implementation were as follows: prescribers' representations on patient education, considered of low value; on oral anticancer therapies, considered too dangerous to be handled by the patient him/herself, the indefinite legitimacy of certain professions in charge of patient education programs; patients' engagement in their care pathway and provision of caregivers.

Conclusion: Recommendations include developing patient education culture within the environment of the medical doctors' curriculum, to consider contextual, pre-existing cooperative units for implementing patient education, to systematically send patients to patient education programs without practicing triage. Successful implementation of patient education critically depends on the prescribing physicians' perceived value of patient education. Patient education should become mandatory, integrated as part of the cancer care pathway. Physicians lack the necessary time and/or means to assess patients' capacity for engagement, without adequate strategies for their support. Therefore, physicians should systematically refer all patients to patient education, where nurses can tailor their coaching of cancer patients.

Trial Registration: The study protocol was approved by the IRB SUD EST I ( ${ }^{\circ}$ EudraCT: 2016-A00113-48). All participants were given written and verbal information about the study and gave informed consent to participate.

Keywords: oral anticancer therapy, implementation science, patient education, theoretical domains framework

\section{Introduction}

Patient education (PE) has demonstrated efficacy and effectiveness in fighting chronic diseases. ${ }^{1}$ Fewer data are available on the assessment of patient education effectiveness for cancer patients and even fewer concerning prescription of oral anticancer therapies, partially due to the novelty of the research in the field. ${ }^{1,2}$ In comparison to results in other fields of other pathologies, like diabetes or asthma, patient education should be an effective way of securing the management of the 
prescription of oral anticancer therapies and supporting cancer patients. The final objective of patient education is to empower patients to live their optimal lives with their disease and their treatment. ${ }^{3}$ Its operative objectives are to increase treatment adherence, improve the management of drug and food interactions, of safe handling and storage/ disposal of the OAT, better offset side effects, and eventually reduce non-scheduled hospitalizations. ${ }^{4-8}$

Oral anticancer therapies for cancer (OAT) are increasingly prescribed. They enable patients to be more independent, reduce the number and duration of hospital admissions and improve their quality of life. $^{4-7}$ Yet patients still need follow-up, most often during hospital consultations (ranging from every 3 weeks to twice a year), which take time for both prescribers and patients. Despite this, these consultations are not always the place for the patient to find the answers to the questions he has about his daily life with the treatment.

Prescribing OAT definitely changes the representations (conceptions, ideas and beliefs) of treatment and disease, not only for patients but also for caregivers. ${ }^{9}$ Three French comprehensive cancer centers decided to collaborate on a project aiming at developing and implementing a $\mathrm{PE}$ program dedicated to cancer patients treated with oral anticancer therapies, adapting to the context of each institution. The educational objectives of the core of the program were to improve treatment adherence, side effects management abilities and management abilities of all the conditions of medication intake for patients treated with oral anticancer therapies for progressive cancer (adjuvant endocrine therapy excluded). Each center had to tailor the program to the local context, without it affecting the fundamental core of the program. Prior to the implementation of the patient education program, healthcare providers (nurses, managers, oncologists and hospital pharmacists) of the institutions were trained i) in patient education; ii) in the core of the program to be able to take ownership of it and iii) in adapting it to the organizational context of their institution and to the characteristics of their patients.

Implementing a PE program that can be transferred and integrated into different contexts implies taking into account numerous pre-existing factors of success or failure. Among them, organizational factors are considered major. ${ }^{10}$ Indeed, hospitals are a milieu of a highly interdisciplinary exchanges. Furthermore, teams may have different representations and different features regarding patient education in oral anticancer therapies, depending on the context, the history of the center and their own backgrounds. Questioning the representations of the caregivers on oral anticancer therapies PE once the implementation phase has been completed will allow us to identify the conditions to be met for the proper dissemination of similar patient education programs in cancer care units. Then, an evaluation of the implementation of the PE program on the different sites was performed and reported here.

The aims of this study were to collect relevant data to implement an oral anticancer therapies PE program, according to its context:

1) Identify barriers and facilitators, as perceived by the health stakeholders of each site, for implementing the program.

2) Identify practices encouraging or hindering the implementation of the PE program.

3) Produce recommendations for the dissemination of the PE program.

\section{Methods}

A standardized patient education program has been developed prior to this study, in order to be implemented in the 3 different settings. This program was constructed according to the recommendations of the Cancer Patient Education Network. ${ }^{19}$ Patients educational needs were elicited from different sources, and a multidisciplinary committee, including experts in education, in methodology, in pharmacy and tailor in oncology constructed the program through a consensus process. Experts were representatives of the 3 centers. The patient education program is described in detail in supplementary appendix. Participants to this study were interviewed after the program has begun to be offered to patients.

\section{Sample Recruitment}

Representatives of each of the 3 sites were encountered, all of them members of the executive medical committee of each center. They provided a list of names of professionals working in the centers, involved in care or teams management, especially in the field of oral anticancer therapies. Depending on the sites, professionals were contacted either directly by the representatives, who personally arranged the appointments, or through an e-mail sent by the representative, cc'd to our team, requesting them to make an appointment with the interviewers. 


\section{Inclusion and Exclusion Criteria}

Participants had to work at one of the three centers of the study. They had to be involved in the care (physicians, nurses, pharmacists) of patients prescribed with oral anticancer therapies for cancer (any type, location or stages) or in the management of the teams involved in care (nurse executive manager, senior officer ...). Participants had to agree to participate in the interview.

\section{Interview Grid}

The purpose of the interview guide was to explore how the implementation of the oral anticancer therapies' education program is experience and felt by the healthcare professionals involved in or concerned by its deployment. The Theoretical Domains Framework (TDF) was used to construct the base of the interview grid. The TDF was developed by Michie et al to describe practices of healthcare professionals and identify elements of practices that should be targeted for the implementation of new recommendations. ${ }^{11,16}$ In our case, the TDF was used to identify elements that either hindered or facilitated the implementation of new modes of care in everyday practice. This framework allowed us to explore barriers to and facilitators of change in healthcare settings and design implementation interventions. ${ }^{12-14}$ Each domain of the TDF represented behavioral determinants, mediators of behavior change. ${ }^{15}$ The TDF was composed of 12 original theoretical domains outlined in an interview guide. The 12 domains: (1) knowledge, (2) skills, (3) social/professional role and identity, (4) beliefs about capabilities, (5) beliefs about consequences, (6) motivation and goals, (7) memory, attention and decision processes, (8) environmental context and resources, (9) social influences, (10) emotion regulation, (11) behavioral regulation, and (12) nature of the behavior. ${ }^{16} \mathrm{~A}$ back and forward translation of the prompt by Michie et al named "Theoretical domains, component constructs, and eliciting questions for investigating the implementation of evidencebased practice" was carried out. ${ }^{16}$ Then, 5 researchers reviewed the French version to ensure a good understanding in French. It was subsequently presented to 5 health professionals to confirm its relevance to the target audience.

In order to complete the data by collecting the issues spontaneously raised by interviewees, a nondirective question: "Could you please tell me about the oral anticancer therapies Patient Education Program in your department?" was added at the beginning of the grid. The form and content of the interview guide were validated by a $\mathrm{PhD}$ medical anthropologist (VRD), specialized in the cancer research field.

\section{Data Collection Phase}

The semi-structured interviews were carried out by two professionals: a nurse MSc (EV), female, PhD student, trained in qualitative research and a professional, trained in social psychology, female. Investigators and participants did not know each other prior to the study. The context of the study was explained to each of the participants. Each interview was conducted in one shot, in an office, face to face, with schedules organized to ensure that the professional was fully available. Confidentiality issues concerning the communication of the results and with regard to their hierarchy were addressed. Their consents were systematically collected and recorded. Interviews were fully recorded on a digital audio recorder. Each interview was transcribed in verbatim and anonymized.

\section{Qualitative Data Analysis}

These data were analyzed, in order to generate themes that could be in the aftermath associated with the Theoretical Domain Framework of behavior change to investigate implementation problems. ${ }^{12,13,15-17}$

Specifically, we applied the methodology framework proposed by Braun, V.and Clarke, V. ${ }^{18}$

1.Familiarization with the data. This phase involves reading and re-reading the data, to become immersed and intimately familiar with its content.

2.Generating initial Codes: this phase involves generating succinct codes that identify important features of the data that might be relevant to answering our research question. It involves coding the entire dataset, and after that, collating all the codes and all relevant data extracts, together for later stages of analysis. Nvivo 11 pro software (QSR International) was used to perform the analyses.

3.Searching for themes: the collection of codes was worked on in pair with the $\mathrm{PhD}$ researcher (VRD) to validate a comprehensive interpretation and a grouping of code elements into themes.

4.The resulting themes were compared to the TDF categories, independently by the 2 researchers, and then compared and discussed. Items that could not be included in the TDF framework were thematized separately.

5.Finally, a more refined coding was used for each part of the verbatim used to illustrate the themes.

The participants provided no feedback on the results at the time, but the results were shared with the coordinator 
Table I Participants Characteristics

\begin{tabular}{|l|l|}
\hline $\mathbf{N}=\mathbf{2 0}$ & $\mathbf{N}(\%)$ \\
\hline Profession & \\
Prescriber (MD) & $4(20 \%)$ \\
Pharmacist & $5(25 \%)$ \\
Nurse manager & $2(10 \%)$ \\
Nurse educator & $2(10 \%)$ \\
Coordination nurse & $5(25 \%)$ \\
PE project manager & $2(10 \%)$ \\
\hline Centres & \\
I & $12(60 \%)$ \\
2 & $4(20 \%)$ \\
3 & $4(20 \%)$ \\
\hline Age & \\
{$[20-45]$ years old } & $11(55 \%)$ \\
{$[46-65]$ years old } & $9(45 \%)$ \\
Female & $16(80 \%)$ \\
Male & $4(20 \%)$ \\
\hline
\end{tabular}

of the multidisciplinary committee in charge of the implementation of the program to be able to detect and account for distortions that might be in the data.

\section{Ethical Statement}

The COREQ checklist guided the reporting of this qualitative study. ${ }^{20}$ Ethical approval for the study was granted by the French Sud-Est IV Ethics Committee, $\mathrm{N}^{\circ}$ EudraCT: 2016-A00113-48.

\section{Results}

Twenty health professionals involved in the implementation of the PE oral anticancer therapies program accepted to be interviewed, out of a total of twenty-four initially suggested. The collection took place from October 2017 to June 2018. Seven semi-structured interviews were carried out by EV and thirteen interviews were conducted by the other interviewer. None of the researchers had any connection with the professionals they met. The average duration of the interviews was 35 minutes (from 10 minutes to 60 minutes).

Table 1 presents the overview of the sample.

\section{Barriers and Facilitators Encountered During PE Program Implementation}

See Table 2 for an overview of the barriers and facilitators encountered in implementing the PE program.
Table 2 Barriers and Facilitators Encountered During PE Program Implementation

\begin{tabular}{|c|c|c|}
\hline & Barriers & Facilitators \\
\hline $\begin{array}{l}\text { According to } \\
\text { the specific } \\
\text { occupations }\end{array}$ & $\begin{array}{l}\text { Prescribers of oral } \\
\text { anticancer therapies } \\
\text { prefer a patient sur- } \\
\text { veillance approach } \\
\text { rather than an edu- } \\
\text { cational approach } \\
\text { - Coordinating nurse's } \\
\text { representation on } \\
\text { their everyday } \\
\text { practice } \\
\text { Prescribers have diffi- } \\
\text { culty considering PE } \\
\text { as a central link in the } \\
\text { support of patients } \\
\text { treated with oral } \\
\text { anticancer therapies } \\
\text { - Prescribers do not } \\
\text { believe in PE, specifi- } \\
\text { cally dedicated to } \\
\text { oral anticancer } \\
\text { therapies }\end{array}$ & \\
\hline $\begin{array}{l}\text { Related to the } \\
\text { global system } \\
\text { mode of } \\
\text { operation }\end{array}$ & $\begin{array}{l}\text { - The lack of transport } \\
\text { support to integrate } \\
\text { a PE program } \\
\text { - The provision of } \\
\text { caregivers for the PE } \\
\text { program, in the } \\
\text { absence of dedicated } \\
\text { staff }\end{array}$ & $\begin{array}{l}\text { - Legitimacy of phar- } \\
\text { macists to take over } \\
\text { PE to strengthen the } \\
\text { city-hospital link as } \\
\text { part of oral antican- } \\
\text { cer therapies } \\
\text { prescriptions } \\
\text { - The legitimacy of the } \\
\text { prescriber, as } \\
\text { a Medical Doctor, to } \\
\text { coordinate the PE } \\
\text { program, from the } \\
\text { perspective of all the } \\
\text { caregivers }\end{array}$ \\
\hline $\begin{array}{l}\text { Declared as } \\
\text { related to } \\
\text { patients' } \\
\text { characteristics }\end{array}$ & $\begin{array}{l}\text { - Patients' engagement } \\
\text { is not constant }\end{array}$ & \\
\hline
\end{tabular}

\section{According to the Specific Occupations}

Barrier I: Prescribers of Oral Anticancer Therapies Prefer a Patient Surveillance Approach Rather Than an Educational Approach

In two centers, prescribers seemingly prefer to refer their patients to another coordinator of care, which involves regular telephone follow-up at home. This management strategy 
aims to monitor and provide support to the patient. They have never referred their patients to the PE program.

Could you just explain a little again to me, what it's about, because actually I didn't know it myself, we weren't there, we weren't interested in it, you know, because ... (S1 Prescriber2)

That's also the difficulty, in terms of visibility. No matter how many times you tell the doctors that there's a therapeutic education program, every time: 'Oh really? There's a therapeutic education program? (S2 Pharmacist2)

I think that the fact that there was the ambulatory medical assistance system does not explain the fact that we are not recruiting, but it may also be a system, it's support. Meaning the nurse calls him, which is very good; the patient says whether he's doing well or not, but it's support, we're supporting the patient, That is he doesn't take an active role like with education, it's a bit contradictory. (S2 Pharmacist2)

Regular monitoring of patients undergoing cancer treatment at home seems to take precedence over the educational approach.

\section{Barrier 2: Coordinating Nurse's Representation on Their Everyday Practice}

Coordinating nurses acknowledge that they do not naturally refer patients to patient education. They prefer a pragmatic approach to problem solving, with an almost instantaneous response to the patient's problem.

It's the prescriber; afterwards, if the prescriber doesn't think of it, after them the nurse will come rushing in, but because there's a nurse clinician who'll say 'careful.' The coordinating nurses will fill the educational void; they're going to call them, tell them, you do that, that, that, they're going to handle the prescriptions, they're doing a remarkable job. (S2 Pharmacist2)

Because we can see clearly in our everyday practice that to take certain treatments that are simple and that are not chemotherapy, it is already quite complicated. And ... you sometimes have to arrange a house visit of a nurse to ensure the treatment is properly taken, you know? So, I think that when it comes to oral chemotherapy, yes, it has to be taken properly, and in my opinion, it may be even necessary to implement things to verify that everything is going well. (S1 Coordinating nurse2)
If they don't have the education program, we're not going to say it's going to be disaster, it's going to happen anyway, there's still possible help, there's us, there's already quite a lot of things that exist, but I see this as a plus .... (S1 Coordinating nurse3)

These coordinating nurses explain that they are all trained in the PE program, so the educational approach is also part of their common practice. They declare this may also explain why they do not direct the patient to a PE program, since they themselves can provide it to patients, according to their needs.

We always do therapeutic education, all the time, all the new patients that we see as a coordinating nurse, we do, we do, we do therapeutic education with everyone, you know, we are all trained in therapeutic education and yes, actually I do therapeutic education ... the aim being to ensure that the patient takes charge of his disease and, as we say, is active in terms of his disease you know, yes, of course, therapeutic education I do it all the time. All the time, not that because we do a lot of coordination and anyway, it's our, it's our role, but we also do therapeutic education, of course, every day. (S1 Coordinating nurse2)

In addition, nurses acknowledge that they generally follow the directions of care given by the prescriber.

\section{Barrier 3: Prescribers Have Difficulty Considering PE as a Central Link in the Support of Patients Treated with Oral Anticancer Therapies}

PE seems to be in conflict with the routine practices of the majority of prescribers. Medical doctors do not appear convinced of the effectiveness of patient education programs.

If $\mathrm{PE}$ in oral chemotherapeutic drugs program and it lasts and becomes something, well, it will take a certain amount of time for it become part of society norms. (S1 Prescriber1)

We have also seen that when we speak at conferences, education, doesn't interest a huge amount of people, you see, therapeutic education is difficult, uh, to, to put into action, to motivate. (S3 Prescriber1)

The problem is that PE (...) is not yet ingrained as an added value for doctors. It's starting to have an initial interest with doctors, an awareness. (S2 Pharmacist2)

Like for fatigue, like for ... ok. Well, probably it'll be useful, it will depend on what will be said, and how it is brought to patients. (S1 Prescriber2) 
Moreover, in the 2 centers where monitoring strategies are favored, caregivers other than Medical Doctors believe that it is the physician's responsibility to refer patients to a PE program. They declare that if the physician refers the patient to the PE program dedicated to oral anticancer therapies, few patients would question this prescriptive guidance from the prescriber. They do believe PE should be an integral part of care, but they do not believe it can be implemented without the prescribers' initiative. Nurses explain that when the physician directs the patient to care, it is very rare that the patient objects. In general, it seems that the patient follows the advice of the physician. They also explain that when the nurse provides the same advice, the patient does not respond in the same way and does not necessarily follow the recommendation.

When it is presented by the oncologist as something that is not really negotiable, even if people always have the right to say no, but when we tell them, that's it, it's a part of treatment protocol, people follow, you know people really, you need really tough people, I find, like even to say no, you need, in any case, people who are well ... faced with a doctor, you say nothing, you know (...)For the most part, even if people are going to say, that's it, people who question treatment and everything, is still quite exceptional. (...) Because we are unequal in the relationship of care. (S1 Coordinating Nurse1)

We have recruitment difficulties, but recruitment is not up to us, it is up to the doctors. (S1 Nurse manager1)

\section{Barrier 4: Prescribers Do Not Believe in PE Dedicated to Oral Anticancer Therapies}

Prescribers are reticent and suspicious of a PE program dedicated to the management of patients treated with oral anticancer therapies. They claim not to know what is being dealt with in these sessions. The specificity and harmfulness of oral anticancer therapies seem to dissuade them from patient education. Furthermore, they are against the fact that PE manages oral anticancer therapies according to a generic vision. For them, only a specific view is possible.

The drawback of a general program that applies to everyone in the end, is that it would have had to subprograms, for (...) there are many possible treatments, which have, with directions and explanations we give to patients, there are also many specific side effects, which are specific to drugs, and even which ... and then the monitoring of treatment depends on the disease. (S1 Prescriber1)

We don't have an anti-cancer therapeutic education program. We are going to have more defined projects on the ... it is going to be fatigue, reading biological results, but the variety of oral anti-cancer drugs with the variety of side effects makes a generalized vision of things difficult. (S2 Prescriber1)

\section{Related to the Global System Mode of Operation} Barrier I: The Lack of Transportation Support to Integrate a PE Program

The lack of financial support for home to hospital transport to participate in the PE program is a major obstacle to patient participation reported by numerous interviewees. All the more so as the patients are tired and diminished by a progressive disease. This is a break in coherence between the decisions of the governance and the lack of structuring on the ground so that the patient can benefit from and access the offer, insofar as he/ she has physical and material possibilities. Doctors who do not perceive the added value of PE pose an additional barrier to potential participation, as they not only do not refer the patient to this type of intervention, but also do not prescribe transportation.

Unlike the others, the patient is at home and one of the major obstacles to therapeutic education is the fact that he has to come back to the site. By definition, the patient is at home so coming back deliberately for a workshop, it seems complicated. Especially when you know that $60 \%$ of our patients live outside the Rhône department (...) but it's just that there is at least 25 miles to drive. (S3 Prescriber1)

We don't have many patients, we have trouble getting them involved, we have trouble getting them to come at their own cost (...) I didn't get this point, but it's a real challenge. In this case, funding for transportation, huge. (S3 Prescriber1)

But ... in the modality itself, I think that's what can be an obstacle for patient. The patients who live downtown- it's easier, the patients who live in the depths of the countryside, I mean, will probably not for 1 hour of education, I mean, because first of all, they do not know what it is. And they are probably not ... able to know that it can bring them something ... And then they will ask for someone to take them there, and for me, I think it's not legitimate to make a transportation prescription for that. (S1 Prescriber2) 


\section{Barrier 2: The Provision of Caregivers for the PE Program, in the Absence of Dedicated Staff}

It seems that the proper implementation of the PE program is definitely linked, from a management point of view, to the goodwill of the supervisors, to their capacity to organize, endorse the project and allocate nurse time in order to make possible the execution of the program, as reported by numerous interviewees, regardless of occupations.

Being able to set aside an hour, an hour and a half during the workday, which is already good ... this is the main constraints ... (S3 Coordinating nurse1)

I had it enormously easy because I had very motivated people, especially health executives, who freed staff for training, and then engaged in the workshops so on. (S3 Prescriber1)

The lack of financial valuation of PE often leads to a constant unsatisfactory deployment in institutions. This is why its continuity often depends on the availability of professionals, the presence of the project manager and the financial resources allocated.

I was on leave for a long time, and so, since it's me who is the pilot of the program.,(...) nobody replaced me in education during that time, so the oral chemo medication activity was not the priority as long as I wasn't there. (S2 Pharmacist2)

Facilitator I: Legitimacy of Pharmacists to Take Over PE to Strengthen the City-Hospital Link as Part of Oral Anticancer Therapies Prescriptions

For sites 1 and 2, given that prescribers do not perceive the added value of referring patients to a PE program, pharmacists were able to seize the occasion to serve their unit's purpose. They welcomed the opportunity to receive funding through the project to train the pharmacy teams in PE, then structure the patient-care pathway with different times of interventions of the pharmacists, in terms of the safety and monitoring of the patient treated with oral anticancer therapies.

The idea is to be able to integrate educational workshops into patient oral chemotherapy care pathway, especially these educational workshops. At the end of the pharmaceutical consultation, we promote the education, specifying that there are not only workshops on treatments, there are other avenues to explore. They are given as much as possible, unless the patient refuses, a consent form that allows us to call them back if they are interested. This truly coherent a continuation of the patient's care treated with oral therapeutics. (S2 Pharmacist3)

It was also a goal that we had, so it actually enabled us ... it just enabled us to implement, to give ourselves the means of our own, allow for us to authorize, in any case to authorize my colleagues, I mean to authorize ... to also allow them to, to be trained. They were things we had too ... It has been the pretext of accepting something that was still within the objectives of the service that everyone be trained in education. And ... it nurtured our pharmaceutical project ... but ... not that it allowed us to say that what we were going to do with this program is something that we needed to take ownership of on a long-term basis, beyond this specific program of education. (S1 Pharmacist 1- Pharmacist 2)

But we also have pharmacists who are extremely committed to it and extremely involved in this project, and who will not easily let go. (S1 Nurse manager1)

Facilitator 2: The Legitimacy of the Prescriber, as a Medical Doctor, to Coordinate the PE Program, from the Perspective of All the Caregivers

At center 3, the oncologist himself is the coordinator of the PE program. Thus, he is legitimized and considered the engine of the project, which is considered a life booster of the team. The whole team is united around the program, and even if some caregivers are not involved in the PE dynamic, no obstacles are mentioned. All patients are referred by this prescriber, to the PE program. The coordinating nurses work alongside the doctor and are very involved in PE. PE is an integral part of the philosophy of patient care in this unit. Other caregivers not invested in $\mathrm{PE}$ are not opposed to it and may also be solicited for their expertise.

So, in the project, in fact, we have reeducators, and thus, dieticians, so the team has actually changed, so the dieticians who have been trained in therapeutic education have left so their colleagues have to take over. So, there is a psychologist who intervenes and also ... an onco nurse .. . it seems to me. So, there you go. But actually, according to the workshops that are proposed, there are 5 workshops, the professionals take on different ones if you want. So, um ... feeling good about your body, for example, is a physical therapist who leads it, who leads that workshop. Nutrition it's also connected with a nurse and the dietician. So, it's a multi-professional team in any case. So that's it. (S3 Nurse manager1) 
We were a team dynamic and there were many of us, being part of these $40 \mathrm{~h}$, both at the nurse and caregiver level. And the nurse manager was conciliatory, in terms of our hours, in fact, because we do the workshops during our working hours. (...) With everyone's willingness it's feasible. (S3 Coordinating nurse1)

On the contrary, regarding center 1 , the team supporting the PE project is not recognized as legitimate by the prescribers, because the team is made of researchers. Despite being Medical Doctors, they are not physicians, and are not considered as such by their oncologist counterparts. There is no notion of belonging to the same team.

... she's not a physician, right, not anymore, so ... (S1

Prescriber2)

\section{Declared as Related to Patients' Characteristics Barrier I: Patients' Engagement is Not Constant}

For a number of professionals, PE cannot concern all eligible patients. They consider that other strategies must therefore be mobilized to ensure that treatment is followed, and taken correctly, without misuse. Moreover, they also report that not all patients are willing to participate in such a program, without them being able to objectively demonstrate this. The issue of the ability to assess the patient's capability to become pro-active is also raised.

There are patients who will be sensitive to therapeutic education, others not ... other patients who are going to prefer to have someone they know, who they have seen throughout the treatment and who will be more connected, me, I have patients who never call the pivot nurse, who call here directly, because they don't want an intermediary, I mean, that's it, after each one is a little different depending on his/her experience. It may be very interesting for certain patients. After which ... it's hard to say, you know? Yes, I think it varies a lot from one patient to another. (S1 Prescriber2)

If the patient doesn't have skills in managing his toxicities, the caregiver team can accompany him or her in various ways, there can be telephone calls by nurses at home. It is even possible to envisage a decompartmentalization of patient care by independently contracted teams, in the city. Afterwards, if there is a failure of his skills, maybe we can try reasons for this failure, perhaps propose other things. (S2 Pharmacist3)

It is important to make an assessment of what the patient is able to do, to assess the autonomy he can have and understanding. (S1 Nurse manager1)

\section{Practices to Enhance or to Hinder Implementing a PE Program Dedicated to Oral Anticancer Therapy}

See Table 3 for an overview of the practices to enhance or to hinder implementing a PE program dedicated to oral anticancer therapy

\section{Practice to Hinder \#I: Construct the Program by Bringing Together PE Experts and Novices}

The construction phase of the program brought together both novices in PE (requiring a 40-hour regulatory training module) as well as experts in PE. In addition, all professional care categories were mixed. But it seems that this approach failed to address issues of professional hierarchy. The doctors allegedly took up a lot of space.

There was multi-disciplinarity, but there was no interdisciplinarity. (...) moreover, things were oddly built, which means that they found themselves in the expert group and people who were in training for2 hours, I mean, it's because they were doctors and perhaps took up a lot of space (...) In other words, it was a monumental mistake, in my opinion, to mix the two. (S1 Project Manager2)

\section{Practice to Hinder \#2: Construct the Program by Excluding Nurse Educators}

PE nurses were not invited to participate in the coconstruction of the program. This created difficulties on the

Table 3 Practices to Enhance or to Hinder Implementing a PE Program Dedicated to Oral Anticancer Therapies

\begin{tabular}{|l|l|}
\hline Practices to Hinder & Practices to Enhance \\
\hline $\begin{array}{l}\text { \#I Construct the program by } \\
\text { bringing together PE experts and } \\
\text { novices }\end{array}$ & $\begin{array}{l}\text { \#I Construct the PE program as } \\
\text { a team cohesion tool, abolishing } \\
\text { hierarchies and allowing the } \\
\text { recognition of the skills of all }\end{array}$ \\
\hline $\begin{array}{l}\text { \#2 Construct the program by } \\
\text { excluding nurse educators }\end{array}$ & $\begin{array}{l}\text { \#2 Be transparent about the fact } \\
\text { that the PE program is a means } \\
\text { to collectively move from } \\
\text { a paternalistic to a patient- } \\
\text { centered approach }\end{array}$ \\
\hline $\begin{array}{l}\text { \#3 Compare and replicate the } \\
\text { project of a previous program } \\
\text { that failed using the same } \\
\text { process }\end{array}$ & \\
\hline $\begin{array}{l}\text { \# Bring together two specialties } \\
\text { that, historically, have failed to } \\
\text { work together }\end{array}$ & \\
\hline
\end{tabular}


ground for them to take ownership of the program. As a result, the tools and modules, which had been designed in advance by other caregivers not directly invested in executing the PE program, were not adaptable or in accordance with what could have been performed on the ground with patients.

Because in fact it was, I had the impression that it was not always applicable in real life, which had a little, a ... yeah a difference between what had been decided during training, in any case what had been imagined and during the workshop ... concretely how we do what, you know? ... That's it, that's a bit what the educational nurses felt, made me feel in any case. (S1 Project Manager1)

What caused problems is more what was provided to them because, on the other hand, none of the girls in the therapeutic education team, meaning none of the trainers who were brought to, leading the project, were invited to lead the expert group training, so it is just an aberration, apart from the team manager, who did not even come all the time by the way, so it's just a huge like whatever, I mean that the actors on the ground are not involved in the construction of the project, it's ... it's nonsense. (S1 Project Manager2)

Practice to Hinder \#3: Compare and Replicate the Project of a Previous Program That Failed Using the Same Process At center 1, the previous failure of a PE program based on a specific molecular scale of oral anticancer therapies was still experienced as a crushing failure. The implementation of the PE program, which was not presented as a brand new one, reinforced the mistrust of prescribers and the coordinating nurse about the value of such a PE program.

The experience we have with the first oral chemo program, the one we had built on Xeloda ... makes me fear certain . . . difficulties (S1 Project manager2)

As for me at the beginning I, well even before this program, we had done stuff on Xeloda. And it was hyper complicated (S1 Coordinating Nurse1)

Practice to Hinder \#4: Bring Together Two Specialties That, Historically, Have Failed to Work Together

In Center 1, the PE program aimed to include both oncology and hematology patients, even though patients were never managed and treated together by the oncologist and the hematologist.
But, even though, in fact ... patients included in public health programs are patients who are monitored by oncologists or hematologists, so ... this is a bit of a problem with public health programs.(...) It is not a program of a hematological research department, so we do not know very well what it's about, how many patients are included ... (S1 Prescriber1)

It seems that the oncology and hematology departments are highly incongruent and have always worked separately. The prescribers mention that it seems extremely difficult to envisage reuniting patients in these two departments within the same oral anticancer therapies PE program.

Here, there really is a dichotomy a bit historical, in the sense that the departments were not together (...) Here, we don't take care of the same patients, you know what I mean, right? (...) in the way in which we care for patients ... we do not have the same way of doing things at all. It's not the same organization at all. (S1 Prescriber2)

Practice to Enhance \#I: Construct the PE Program as a Team Cohesion Tool, Abolishing Hierarchies and Allowing the Recognition of the Skills of All

At center 3, the deployment of the PE program has enabled some caregivers to demonstrate in their skills. The team unanimously explains that through the implementation of this PE program, all the professionals invested in the program are valued in their role and skills, without any hierarchical distinction.

Me, I think that's what I'm saying, you know, that it's really it's tool for recognizing the staff for their jobs (S3 Prescriber1)

We become employees, I think it's important, it's ... we don't have the, a hierarchical role or I don't know what, it's really what we are, we're really employees and that's it, I think it's very positive (S3 Prescriber1)

Practice to Enhance \#2: Be Transparent About the Fact That the PE Program is a Means to Collectively Move from a Paternalistic to a Patient-Centered Approach

At center 3, collectively building the PE program has had the effect of completely changing the patient-care givers relationship as it could have historically been: in a paternalistic way. Since the implementation of this project, the relationship between patients and care givers have been perceived by all as complementary and symmetrical. This allowed caregivers to open up to another dimension of care, with a paradigm shift: the emergence of patientcentered care. 
Patient Education is still for the patient and then for us, it has enabled us, the caregiver, in any case, to dig a little more into our knowledge and to update it and renew it progressively for the patient. So, I think it is a good thing for them and for us. It's really ... and for the caregiver and for the patient. (S3 Coordinating nurse2)

With education, what there is, is that you still take care of the patient as a whole and I think it's important, it's not a disease you're targeting, it's not a treatment you're targeting, but it's really a whole person. So, that, I think it has its value in the therapeutic education program. (S3 Coordinating nurse2)

It is no longer the hospital that is all-powerful, it is no longer we who are all-powerful. It's, um ... it's the patient who's also an actor and us, we pull back a little bit, we are, we are there to guide them. Then, at times, it is them who actually guide us. So, it is a role of exchange, which is ... which is actually new. But which is, the future I think of the hospital (S3 Coordinating nurse1)

\section{Recommendations Tracks for the Dissemination of a PE Program Dedicated to Cancer Patients Undergoing Oral Anticancer Therapies}

Successful implementation of PE, particularly in oral anticancer therapies, is a complex intervention. To benefit from recommendations aimed at successfully implementing such a program would be an asset for all other cancer treatment centers in France. The US National Cancer Institute Patient Education Guidelines are designed to serve as a model to help cancer centers, hospitals, clinics and teaching institutions to develop and improve the delivery, management and quality of their cancer patient education services. ${ }^{21}$ We have merged our results with those guidelines in order to produce some ground rules of reflection. We provide six additional tracks to consider before implementing a PE program dedicated to oral anticancer therapies (based on our results).

See Table 4 related to the Recommendations tracks for the dissemination of a PE program dedicated to cancer patients undergoing oral anticancer therapies

\section{Discussion}

This set of interviews leads us to better understand several issues related to a patient education program implementation in oral anticancer therapies setting:

- The stakeholders should already be used to working together for existing care, with common
Table 4 Recommendations Tracks for the Dissemination of a PE Program Dedicated to Cancer Patients Undergoing Oral Anticancer Therapies

I.The PE has a statement of mission and a statement of philosophy which concisely identify the purpose and reflect the value of the program $^{19}$

In order for a PE program in oral anticancer therapies to be implemented and to be functional, statements setting out the philosophy and organization of patient education must be made at the institutional level. These declarations must be shared and their values instilled in all professionals of the establishment.

2.The PE staff are empowered and accountable for administering patient education programs and activities: written rules attest that it is the staff of the PE program who decide, who is responsible for its development, implementation, coordination and evaluation. ${ }^{19}$

Co-construction of a PE program must include the entire team of stakeholders. In addition, institutional support would make it possible to legitimately position stakeholders in the eyes of all professionals with regard to the implementation of a new intervention, a new project.

3.The stakeholders should already be used to working together for existing care, with common processes and common cultures, before implementing a shared patient education program

4.The patient education coordinator should belong to the care team, and be a key opinion leader inside the team

5.Patient empowerment culture should be widespread to include all healthcare workers' curriculum

6.Medical Doctors prescribers should be sensitized and trained during their courses in patient education. They are the cornerstone of the patient pathway and adherence

7.Patient education should be mandatory care, integrated care as part of the cancer care pathway, mandated and facilitated by the National Health System

8.Patients should systematically be directed to the patient education program, regardless of the impression the care givers may have of the patient's willingness to participate or regardless of the care givers detrimental beliefs towards patient education

9. Communication based on a patient education program should be handled with care and a professional approach: communications to other caregivers, managers and patients have different ways and purposes

processes and common cultures, before implementing a shared patient education program

As Jaffiol et al pointed out in 2013, our results highlight the importance of sharing a common culture in project 
management to achieve the objectives and successfully implement the new intervention. Jaffiol et al explained that establishing a common culture of $\mathrm{PE}$ is essential to fostering multi-professional cooperation in the management of patients with chronic diseases. ${ }^{21}$ Indeed, we observed that the results of the implementation were very different depending on the centers. Site 3's team is federated with the program everyone shares the philosophy of PE so the program is implemented completely. At sites 2 and 3, prescribers are not particularly in favor of PE. Pharmacists, however, fully adhere to PE. The pharmacy teams of these 2 sites share the philosophy of PE and have therefore structured and implemented one workshop dedicated to their specialty (drugs). The program is only partially deployed at site 2 , and inexistent at site 1 .

- The patient education coordinator should belong to the care team, and be a key opinion leader inside the team

The legitimacy of the PE program coordinator is central to its proper implementation. In fact, on the 2 sites ( 2 and 3 ) where the coordinator was part of the medical team, the project was able to be carried out (even partially). At the site where the program coordinator was not part of the medical team, the program aborted. We can link the recommendations made by Heins et al to the multi-center implementation of a PE program in diabetology. The visible involvement of esteemed physicians or other health professionals respected by their peers was stated as a prerequisite for successful implementation and sustainability of the program. ${ }^{22}$

- Patient empowerment culture should be widespread to involve all healthcare workers' curriculum

As we saw through the results, the implementation of the program was very different depending on the sites. For site 1 , despite a team dedicated to patient education, with all the necessary resources, the cultural context combining oncology and hematology, the lack of legitimacy of the program coordinator and the reluctance of prescribers to empower the patient treated with oral anticancer therapies led to abortive implementation. At 3 , in contrast, despite a lack of resources, the multidisciplinary team is driven, bringing together a common project that makes sense to all. Despite the difficulties, the PE program was implemented. Finally, at center 2 , the situation is intermediary. The pharmacy team is the determined group, motivated to integrate PE into the cancer care pathway as part of the management of oral anticancer therapies. Despite the lack of buy-in by prescribers to the PE program, they do not oppose it. Sites 1 and 2 highlight the difficulty of physicians and nurses adhering to the Patient's Educational approach. Those who do not adhere to PE, leave this prerogative to pharmacists, specialists in the molecule and its effects, without referring patients to a PE program. The Federation of Haematological/ Oncological Pharmacy shows the important role of oncology pharmacists in teams managing cancer patients. ${ }^{23}$ Paolella et al stress the importance of the pharmacist in this coaching process. The drug specialist is able to identify drug problems before, during, and after beginning oral anticancer therapies. Treatment adherence, medication changes, and medication tolerance in a cancer patient are dynamic variables that require special attention and increased communication with the healthcare team. ${ }^{23}$ Pharmacy team has a transversal and well-recognized function inside institutions. This leads to a structural and collaborative partnership with all others departments and units involved in the patients care. This institutional position could ensure the acceptability by all of a patient education program promoted by this team. Such organization could be supported on the condition that pharmacists feel comfortable and qualified to do so.

Shifting the burden and responsibility for the disease and its treatment from the caregivers to the patients and letting them manage themselves seems unbearable to the caregivers. All the more so if the severity of the condition or treatment is considered consequential. The situation is analogous to the phenomenon experienced by parents when their children leave home: the empty nest syndrome. As Edith Goldbeter-Merinfeld analyzed these "crisis" families, where parents and young adults have difficulty separating, "the way in which the period of adolescence is lived, or becomes stuck, is also linked to the empowerment patterns of earlier generations". ${ }^{24}$ This phenomenon of denial of the autonomy of the other, with the virtuous but misguided aim of protection, indeed challenges the self-empowerment capacity of current caregivers. It is this observation that has led us to make the last recommendation.

- Medical Doctors prescribers should be sensitized and trained during their curriculum in patient education. They are the cornerstone of the patient pathway and adherence 
However, the PE and its culture positions the patient at the center of the care system, across all its lifestyles, which contradicts the historic hospital culture. L. Gerbaud explained that until recently, the institutions were the heart of the healthcare system, and positioned themselves as responsible for the patient. In contrast, he underlines that the principles of PE reflects an other process that aims to place the patient at the centre of the healthcare system, as an active subject. ${ }^{25}$ Bourmaud et al have shown that patients treated with oral anticancer therapies are asking for tailored educational interventions, driven by their healthcare providers. ${ }^{26}$ It is therefore an opportunity to put the patient back at the heart of care, in the institution, by modifying professional Thought software and returning to a symmetrical care-care relationship. Everyone will be able to recognize and take advantage of the expertise of the other: the expert patient in his or her construction, based on his or her experiences and experience; the expert caregiver in his or her care-taking practice. The results of our research showed that when an oncologist is convinced and involved in PE, the whole team becomes engaged in its implementation. PE becomes not only a driver for the service of care, but also a way to switch from the old paradigm, which is only prescriptive and paternalistic, to a paradigm where care becomes patient-centered, with an individualized and holistic approach.

$\mathrm{PE}$ is involved in supporting patient empowerment and a patient-centered approach. This approach is one of the new objectives of French health policy, unlike other countries where these methods of care have long been adopted. $^{27,28}$ According to Härter et al, France is poorly classified in terms of patient-centered care. ${ }^{27}$ The analysis shows that most doctors and nurses have difficulty adhering to the educational approach, which calls for abandoning the paternalistic, prescriptive and patient-monitoring posture. A previous study by Verot et al corroborates these findings, showing that cancer caregivers find it difficult to change their care practices and strategies in support of the empowerment of cancer patients. ${ }^{29}$ The patient, in hospital culture, is passive and abandons him/herself (consciously or not) to the care of hospital caregivers. This was explained in a report on a coordinated research program focused on PE between 2012 and 2015, in several French hospitals. This intervention research sought to deploy PE in the different institutions and then explore its effects. It explains that the culture of care that is taught centered on technical cares. In the context of organizational difficulties, patient management focuses on the technical aspects of care, sometimes even dehumanizing the patient. $^{30}$

- Patient education should be mandatory integrated care as part of the cancer care pathway, mandated and facilitated by the National Health System (NHS)

Evans et al recall that integrated care encompasses not only the notion of organization, but also the notion of patient-centeredness. Cancer patients are complex and vulnerable. Physicians and nurses should provide cancer patients integrated care in hospitals. ${ }^{31}$ In a recent study of integrated care, Auschra isolated a number of barriers to integrated care in the institution at its various levels: siloed work, different professional cultures, a lack of trust, different objectives, a lack of stable leadership or coordination, and political arbitrations - all obstacles that we found in our analysis. ${ }^{32}$ The leverages addressing those issues must be reinforced at the systemic level.

If PE were officially integrated into the patient-care pathway treated with oral anticancer therapies, this would allow:

\section{- Communication involving a patient education program to be handled with care and a professional approach}

Thus, communications to other caregivers, managers and patients have different ways and purposes. Prescribers, General Practitioners, liberal nurses and city-pharmacists can also be relays that promote a cohesive discourse and care. $^{33}$

- Patients should systematically be directed to the patient education program corresponding to their condition, regardless of the impression the caregivers have or not of their willingness to participate or regardless of the care givers detrimental beliefs towards patient education

Zerillo et al emphasize the need for a care pathway that would reassure patients treated with oral anticancer therapies. ${ }^{34}$ The prescriber's power of persuasion as regards to patient education has a major impact on patient adherence, which explains why patient acceptance of the PE programs is lower when the educational team is presented to patients as independent of the "classical" care pathway. On the contrary, 
if all healthcare providers are benevolent, coherent and systematics, this consistency will be a major enhancer to patient adherence. ${ }^{31}$ Several healthcare professionals have mentioned that not all patients can become proactive in managing their disease and treatment. But physicians do not have the time, the means or the capabilities to assess patients' capacity for engagement, nor the type of strategies to determine their support. Therefore, physicians should routinely refer all patients to $\mathrm{PE}$, and nurses will be able to tailor the assessment and coaching of cancer survivors.

\section{Limitations of the Research}

A possible selection bias could have been performed through a non-homogeneous and nonrepresentative sample, especially in terms of the professional categories. The small size of the sample cannot be used to extract representative quantitative statistical data due to lack of power. However, the sample size is compatible with reliable qualitative analysis. ${ }^{35}$ The complexity of the implementation process could not have been explored with any other methodology.

\section{Conclusion}

Successful implementation of a PE program dedicated to oral anticancer therapies relies on the establishment of a common culture and a process common to all stakeholders, prior to its implementation. The program coordinator must be a team member and a driving force. All healthcare professionals, and moreover, specifically Medical Doctors, should be acculturated to PE and to patient' empowerment, since they are the cornerstone of the patient pathway and adherence. PE must be mandatory integrated care, integrated as part of the cancer care pathway and the French NHS must facilitate its implementation in the field, particularly with regard to access to opportunities for patients residing at home.

Finally, the paradigm shift in cancer treatment facilities is essential in France, in order to be able to reposition patients at the center of their care, enabling them to benefit from all the care strategies that support their empowerment. Such an endeavor implies a complex evaluation of the patient's environment and capacities, in order to develop a tailored care pathway. This could become an important expected duty of advanced practice nurses.

\section{Contributions to the Literature}

- In this research field where few is known, barriers were identified that jeopardize patients access to patient education (PE): barriers related to medical doctors' negative representations towards $\mathrm{PE}$ and system failure to assign a leader of opinion to the head of patient education programs.

- Oral anticancer therapies' field generates stress on the prescribers who are afraid of losing control on their patients, who are considered by them as particularly fragile, whether it's real or not.

- This study proposes recommendations for successful implementation, according to the specific context of a team wishing to deploy PE in the field of oral anticancer therapies.

\section{Abbreviations}

PE, patient education; OAT, Oral Anticancer Therapies; TDF, Theoretical Domains Framework.

\section{Data Sharing Statement}

All data generated or analyzed during this study are included in this published article.

\section{Ethical Approval}

All procedures performed in studies involving human participants were in accordance with the ethical standards of the institutional and/or national research committee and with the 1964 Helsinki declaration and its later amendments or comparable ethical standards. The study protocol was approved by the IRB SUD EST I (N EudraCT: 2016A00113-48).

\section{Informed Consent}

Informed consent, including consent for publication of anonymized verbatims, was obtained from all individual participants included in the study.

\section{Acknowledgments}

The authors would like to thank Olivia Miyataki-Pellarin ("Aloha Language Consulting inc*") for translation and editing services.

\section{Author Contributions}

All authors contributed to data analysis, drafting or revising the article, have agreed on the journal to which the article will be submitted, gave final approval of the version to be published, and agree to be accountable for all aspects of the work. 


\section{Funding}

This study was funded by Institut National du Cancer/ supported by the French National Institute of Cancer (INCa 2014-202).

\section{Disclosure}

Claire Falandry reports personal fees from TEVA, during the conduct of the study; personal fees from TEVA, PFIZER, ASTRA-ZENECA, NOVARTIS, BAXTER, JANSSEN, LEO Pharma, and CHUGAI, outside the submitted work. Catherine Rioufol reports personal fees from BMS, Lilly, takeda, celgene, abbvie, gilead, and novartis, outside the submitted work. The authors report no other potential conflicts of interest for this work.

\section{References}

1. Coster S, Norman I. Cochrane reviews of educational and self-management interventions to guide nursing practice: a review. Int J Nurs Stud. 2009;46(4):508-528. doi:10.1016/j.ijnurstu.200 8.09.009

2. Arthurs G, Simpson J, Brown A, Kyaw O, Shyrier S, Concert CM. The effectiveness of therapeutic patient education on adherence to oral anti-cancer medicines in adult cancer patients in ambulatory care settings: a systematic review. JBI Database Syst Rev Implement Rep. 2015;13(5):244-292. doi:10.11124/jbisrir-2015-2057

3. World Health Organization. Therapeutic patient education: continuing education programmes for health care providers in the field of prevention of chronic diseases - Report of a WHO Working Group. 1998; 77. doi:10.1016/j.ancard.2011.09.006

4. Walko CM, Lindley C. Capecitabine: a review. Clin Ther. 2005;27 (1):23-44. doi:10.1016/j.clinthera.2005.01.005

5. Faithfull S, Deery P. Implementation of capecitabine (Xeloda ${ }^{\circledR}$ ) into a cancer centre: UK experience. Eur J Oncol Nurs. 2004;8 (SUPPL):1. doi:10.1016/j.ejon.2004.06.009

6. DeMario MD, Ratain MJ. Oral chemotherapy: rationale and future directions. J Clin Oncol. 1998;16(7):2557-2567. doi:10.1200/ JCO.1998.16.7.2557

7. Van Cutsen E, Twelves C, Cassidy J, et al. Oral capecitabine compared with intravenous fluorouracil plus leucovorin in patients with metastatic colorectal cancer: results of a large Phase III study. J Clin Oncol. 2001;19(21):4097-4106. doi:10.1200/jco.2001.19.21.4097

8. Twelves C, Boyer M, Findlay M, et al. Capecitabine (Xeloda ${ }^{\mathrm{TM}}$ ) improves medical resource use compared with 5-fluorouracil plus leucovorin in a phase III trial conducted in patients with advanced colorectal carcinoma. Eur J Cancer. 2001;37(5):597-604. doi:10.1016/S0959-8049(00)00444-5

9. Marsé H, Van Cutsem E, Grothey A, Valverde S. Management of adverse events and other practical considerations in patients receiving capecitabine (Xeloda ${ }^{\circledR}$ ). Eur J Oncol Nurs. 2004;8(SUPPL. 1):S16S30. doi:10.1016/j.ejon.2004.06.006

10. Proctor EK, Powell BJ, McMillen JC. Implementation strategies: recommendations for specifying and reporting. Implement Sci. 2013;8:1. doi:10.1186/1748-5908-8-139

11. Cane J, O'connor D, Michie S Validation of the Theoretical Domains Framework for Use in Behaviour Change and Implementation Research 2012. Available from: http://www.implementationscience. com/content/7/1/37. Accessed April 5, 2019.
12. Atkins L, Francis J, Islam R, et al. A guide to using the Theoretical Domains Framework of behaviour change to investigate implementation problems. Implement Sci. 2017;12(1):77. doi:10.1186/s13012017-0605-9

13. Michie S, Atkins L, West R. The behaviour change wheel. A guide to designing interventions; 2014. Available from: http://www.beha viourchangewheel.com/. Accessed October 21, 2020.

14. Michie S, van Stralen MM, West R. The behaviour change wheel: a new method for characterising and designing behaviour change interventions. Implement Sci. 2011;6(1):42. doi:10.1186/1748-59086-42

15. Glasper A, Rees C. Nursing and Healthcare Research at a Glance. Wiley; 2016.

16. Michie S, Johnston M, Abraham C, Lawton R, Parker D, Walker A. Making psychological theory useful for implementing evidence based practice: a consensus approach. Qual Saf Health Care. 2005;14 (1):26-33. doi:10.1136/qshc.2004.011155

17. Phillips CJ, Marshal AP, Chaves NJ, et al. Experiences of using the Theoretical Domains framework across diverse clinical environments: a qualitative study. J Multidiscip Healthc. 2015;8:139-146. doi:10.2147/JMDH.S78458

18. Braun V, Clarke V. Using thematic analysis in psychology. Qual Res Psychol. 2006;3(2):77-101. doi:10.1191/1478088706qp063oa

19. Guidelines for Establishing Comprehensive Cancer Patient Education Services.

20. Tong A, Sainsbury P, Craig J. Consolidated criteria for reporting qualitative research (COREQ): a 32-item checklist for interviews and focus groups. Int J Qual Health Care. 2007;19(6):349. doi:10.1093/intqhe/mzm042

21. Jaffiol C, Corvol P, Reach G, Bas-Devant A, Bertin É L'éducation Thérapeutique Du Patient (ETP), Une Pièce Maîtresse Pour Répondre Aux Nouveaux Besoins de La Médecine L'expansion.; 2013. http://www.academie-medecine.fr/wp-content/uploads/2014/ 01/jaffiolRapport-ETP-voté-10-XII-13-3.pdf.

22. Heins JM, Nord WR, Cameron M. Establishing and sustaining state-of-the-art diabetes patient education programs: research and recommendations. Diabetes Educ. 1992;18(6):501-508. doi:10.1177/014572179201800609

23. Paolella G, Boyd A, Wirth S, et al. Adherence to oral anticancer medications: evolving interprofessional roles and pharmacist workforce considerations. Pharmacy. 2018;6(1):23. doi:10.3390/ pharmacy6010023

24. Goldbeter-Merinfeld E. Adolescence: de la crise individuelle à la crise des générations. Cahiers Critiques de Therapie Familiale et de Pratiques de Reseaux. 2008;40(1):13-26. doi:10.3917/ ctf.040.0013

25. Gerbaud L. Éducation thérapeutique et santé publique: questions croisées. Annales Médico-psychologiques, revue psychiatrique. 2015;173(1):106-112. doi:10.1016/j.amp.2014.12.011

26. Bourmaud A, Henin E, Tinquaut F, et al. Adherence to oral anticancer chemotherapy: what influences patients' over or non-adherence ? Analysis of the OCTO study through quantitative-qualitative methods. BMC Res Notes. 2015;8(1):291. doi:10.1186/s13104-015-1231-8

27. Härter M, Moumjid N, Cornuz J, Elwyn G, van der Weijden T. Shared decision making in 2017: international accomplishments in policy, research and implementation. $Z$ Evid Fortbild Qual Gesundhwes. 2017;123-124:1-5. doi:10.1016/j.zefq.2017.05.024

28. Ministère des Solidarités et de la Santé.Stratégie nationale de santé 2018-2022. Ministère des Solidarités et de la Santé; 2018. https:// solidarites-sante.gouv.fr/IMG/pdf/dossier_sns_2017_vdefpostconsult.pdf. Accessed October 21, 2020.

29. Verot E, Régnier-Denois V, Feld D, Rivoirard R, Chauvin F. French Health professionals' attitudes about patient empowerment in the cancer care pathway. Clin Nurs Stud. 2019;7(4):41. doi:10.5430/cns. v7n $4 \mathrm{p} 41$ 
30. Obertelli P, Pouteau C, Haberey-Knuessi V, et al. Évolution Des Relations Soignants-Malades Au Regard Des Pratiques d'éducation Thérapeutique.; 2015. Available from: https://halshs.archivesouvertes.fr/halshs-01288026. Accessed May 14, 2019.

31. Evans JM, Matheson G, Buchman S, et al. Integrating cancer care beyond the hospital and across the cancer pathway: a patient-centred approach. Healthcare Quart. 2015;17:28-32. doi:10.12927/ hcq.2014.24006

32. Auschra C. Barriers to the integration of care in inter-organisational settings: A literature review. Int $J$ Integr Care. 2018;18:1. doi:10.5334/ijic.3068
33. Ruddy K, Mayer E, Partridge A. Patient adherence and persistence with oral anticancer treatment. CA Cancer J Clin. 2009;59(1):56-66. doi:10.3322/caac. 20004

34. Zerillo JA, Goldenberg BA, Kotecha RR, Tewari AK, Jacobson JO, Krzyzanowska MK. Interventions to improve oral chemotherapy safety and quality. JAMA Oncol. 2018;4(1):105. doi:10.1001/ jamaoncol.2017.0625

35. Saldana J. Cap 2. The Coding Manual for Qualitative Researchers. 3rd ed. sage; 2009. Available from: https://study.sagepub.com/salda nacoding3e. Accessed October 21, 2020.

\section{Publish your work in this journal}

Patient Preference and Adherence is an international, peer-reviewed, open access journal that focusing on the growing importance of patient preference and adherence throughout the therapeutic continuum. Patient satisfaction, acceptability, quality of life, compliance, persistence and their role in developing new therapeutic modalities and compounds to optimize clinical outcomes for existing disease states are major areas of interest for the journal. This journal has been accepted for indexing on PubMed Central. The manuscript management system is completely online and includes a very quick and fair peer-review system, which is all easy to use. Visit http:// www.dovepress.com/testimonials.php to read real quotes from published authors. 